\title{
APPLICABILITY OF NANOPARTICLES-HYDROGEL COMPOSITE IN TREATING PERIODONTAL DISEASES AND BEYOND
}

\begin{abstract}
NAFIU AMINU ${ }^{1,2 *}$, SEOK-MING TOH ${ }^{1}$
${ }^{1}$ Discipline of Pharmaceutical Technology, School of Pharmaceutical Sciences, Universiti Sains Malaysia, 11800 USM, Penang, Malaysia. ${ }^{2}$ Department of Pharmaceutics and Pharmaceutical Microbiology, Faculty of Pharmaceutical Sciences, Usmanu Danfodiyo University,
\end{abstract} P.M.B. 2346, Sokoto, Nigeria. Email: nabgus@yahoo.com

Received: 16 October 2016, Revised and Accepted: 26 October 2016

ABSTRACT

Nanoparticles (NPs)-hydrogel composite (nanogels) have yielded a surge in the design and development of novel drug delivery systems for the treatment of many ailments, including periodontal disease. The recent innovations in nanotechnological drug carrier systems seem promising, as it provides a means to improve the bioavailability of poorly soluble drugs, formulations of controlled and targeted drug delivery systems, and drug release control based on the stimuli response, among others. Several polymeric NPs-hydrogel coformulations have been investigated during the last few years, mostly using synthetic and natural polymers. Some of the results and rewards achieved from these novel approaches are the use of bioadhesive polymers to achieve prolonged drug release, the increment of intra-pocket drug penetration, the enhancement of mechanical properties using chemical crosslinkers, and the possibility of loading multiple drugs in a unit delivery system. Furthermore, these nanotechnological advances have also shown that NPs possess great potential as drug carriers in periodontal disease treatment. The future utilization of these advantages will significantly improve dental care. The coformulation of NPs-hydrogel composite will yield additional benefits that are much greater than ordinary NPs or hydrogels in delivering of drug into the periodontal pockets. The aim of this review article is to summarize updates on the current and future nanotechnological approaches that are being investigated for the treatment of periodontitis, with particular attention to the nanogels, and to identify arenas which its exploration might lead to the development of effective intra-pocket drug delivery systems for the treatment of periodontal diseases. The review also provides brief applications of nanogels in the management of other diseases.

Keywords: Nanocomposite, Hydrogels, Nanoparticles, Nanogels, Periodontal intra-pocket drug delivery system, Nanotechnological approaches.

(c) 2017 The Authors. Published by Innovare Academic Sciences Pvt Ltd. This is an open access article under the CC BY license (http://creativecommons. org/licenses/by/4. 0/) DOI: http://dx.doi.org/10.22159/ajpcr.2017.v10i2.15709

\section{INTRODUCTION}

Periodontitis is a dental inflammatory disorder affecting the surrounding structures of the teeth (periodontium). The disease is characterized by inflammation and destruction of the teeth's surrounding and supporting tissues, i.e., periodontal ligament (PDL), gum, alveolar bone, and cementum which usually occur as a result of anaerobic gram-negative bacterial invasion of the teeth [1].

A diagrammatical comparison of healthy and diseased tooth is depicted in Fig. 1. In the early stage of periodontitis, microorganisms adhere and multiply on the tooth's surfaces which lead to the deposition of plaque and calculus. In the late stage of the disease, the collagen which supports the periodontium will be degraded; the gingival epithelium will shift against the tooth surface, and significant resorption of alveolar bone occurs resulting in the formation of a periodontal pocket [2]. If not properly treated, the consequence of that, along with the body's over-aggressive immune response against the microbes would lead to the destruction of the periodontium and hence loosening and loss of teeth $[3,4]$.

Delivering therapeutic agents to the periodontal pocket (target site) has been a major hurdle in the treatment of periodontitis. Most conventional local drug delivery systems have demonstrated poor results following their administration, due to poor penetration of the junctional epithelium (a circular arrangement of epithelial cells occurring at the base of the gingival sulcus and attached to both the tooth and the subepithelial connective tissue) [5]. Systemic antibiotics are now seldom used for the treatment periodontitis because of drawbacks such as gastrointestinal intolerance, rapid decline of antibiotic concentration in the plasma to subtherapeutic levels, inadequate antibiotic concentration at the site of the periodontal pocket, development of microbial resistance, and hypersensitivity [2]. Similarly, conventional local drug delivery systems are characterized by limited effectiveness, poor biodistribution, and lack of selectivity [6]. These drawbacks have drawn researchers' interest in the development of new efficacious local drug delivery systems for the treatment of periodontitis.

An important positive outcome comes through recent advancement and innovations in nanotechnology, whereby nanotechnology-based colloidal drug carriers could be used to provide site-specific or targeted drug delivery, combined with optimal drug release profiles. Studies have shown that NPs may be a potential carrier system for the delivery of active substances to the periodontal pocket [7]. The incorporation of NPs into polymeric hydrogels has resulted in another interestingly novel class of dosage form that is called nanoparticles (NPs)-hydrogel coformulation (nanogels).

Nanogel delivery systems (Fig. 2) have attracted attention in recent years as one of the most promising nanoparticulate drug delivery systems owing to their unique potentials of yielding beneficial features of NPs (smaller size, site specificity, high stability, and high carrier capacity) and that of hydrogel system (i.e., hydrophilicity and extremely high water content) in a unit delivery system.

The aim of this review article is to acquaint the researchers, formulation scientist, and health-care providers, with the updates on the current and future nanotechnological approaches that are being investigated for the treatment of periodontitis and other ailments, with particular attention to nanogel delivery systems, owing to its unique potentials of achieving multidrug therapy and providing beneficial characteristics of NPs and that of hydrogel system in a unit delivery system. The 


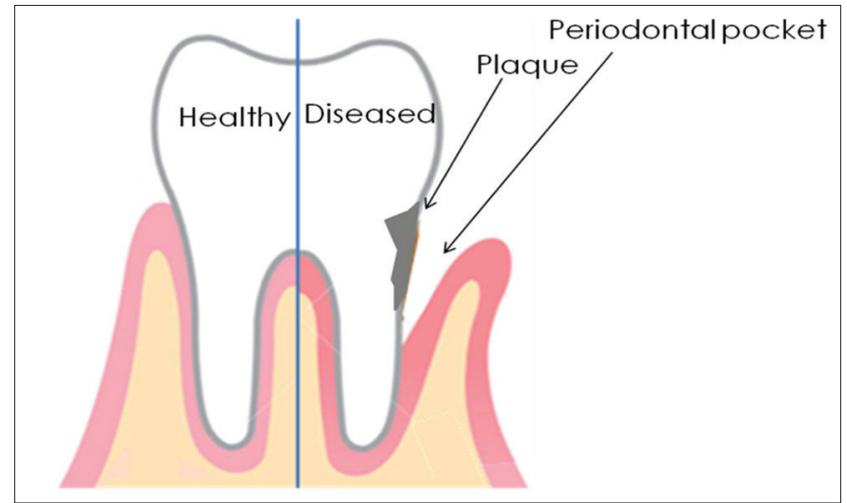

Fig. 1: A comparison between healthy and diseased tooth. Periodontal pocket has developed on the diseased tooth

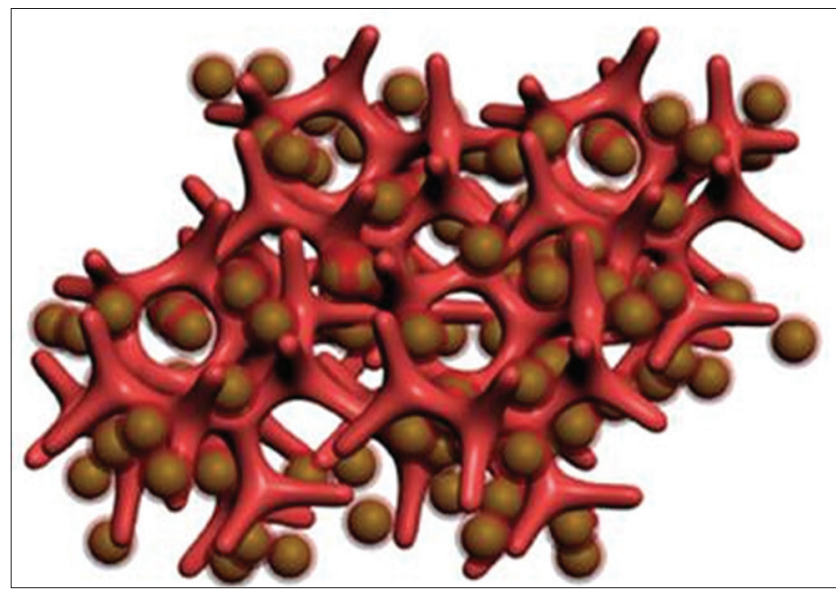

Fig. 2: Schematic illustration of nanoparticles-hydrogel coformulation (nanogels) in 3D [8]

review also aims to identify areas where further research may lead to an effective development of intra-pocket drug delivery systems for the treatment for periodontitis.

\section{NANOTECHNOLOGY IN DISEASE THERAPY}

A significant number of locally administered drug delivering devices have been investigated and proposed over the last three decades (Table 1), but the restriction in accessing the periodontal pocket due to the complexity in anatomy of the root and the contours of the lesion which leads to poor penetration of junctional epithelium by some of these systems has limited their effectiveness in the treatment of periodontitis.

However, nanotechnology may revolutionize preventive, diagnostic, and restorative dentistry by providing nanosized materials that could effectively treat many dental disorders including periodontitis $[34,35]$. Several polymeric NPs-hydrogel coformulations have been proposed in the recent time, using both natural and synthetic polymers and these comes with a lot of advantages (Table 2) over the conventional drug delivery systems. Researchers have studied the transport of NPs through the junctional epithelium and reported that NPs can be a potential intra-pocket carrier system for the delivery of active substances to the periodontal pocket [7].

\section{Nanogels in periodontitis}

The potentials of nanogel delivery systems have been ascertained by many researchers in recent times. It has been reported that nanocomposite biocompatible hydrogels could be used as a model system for in situ local drug delivery devices for the treatment of periodontal diseases. Researchers have developed nanogels consisting of NPs and matrix gel loaded with chlorhexidine for antibacterial effect. They prepared NPs by free radical initiated copolymerization of the monomers and then used the same monomers to prepare crosslinked matrices by photopolymerization. Finally, they developed nanogels by blending the NPs, monomers, and drug in an aqueous solution and then crosslinked it by photopolymerization. This integrated gel system demonstrated distinct advantages compared to simple NPs or hydrogels as a delivery system, and hence, provides the chance of flexible intra-pocket drug delivery in periodontal disease treatment [41].

The PDL cells are essential factors that serve an important role in the regeneration of periodontal tissue, and there is a significant number of investigations that have been carried out to establish and validate its key characteristic features and functions [42]. The recent studies that have been carried out to reverse the periodontal degeneration (a condition that occurs as a result of severe periodontitis) using the transplantation of PDL cells have shown promising outcomes [43,44]. To verify the process of regeneration, Fukui et al. investigated whether cholesterol-bearing pullulan modified with amino groups $\left(\mathrm{CHPNH}_{2}\right)$ nanogels could be used as a carrier to introduce quantum dots (QDs) into the PDL cells that were obtained from a primary culture. The $\mathrm{CHPNH}_{2}$ nanogels make complexes with QDs that resulted in monodisperse hybrid NPs, and it was able to be delivered into live cells by endocytosis, in significant quantity. The $\mathrm{CHPNH}_{2}$ nanogels were found to be useful as a carrier to introduce QDs into PDL cells under culture, and it demonstrated feasibility for further characterization of PDL cells as well as investigation of regenerative processes. This feature further demonstrates the capacity of nanogels system in periodontal therapy [45].

In another related research conducted by Popa et al., chitosan gel containing two antibacterial drugs (tetracycline hydrochloride and metronidazole benzoate) were fabricated for local intra-pocket drug delivery for the treatment of periodontal disease. Based on the kinetic release data and the rheological studies conducted on the periodontal gels, the researchers concluded that $3 \% \mathrm{w} / \mathrm{w}$ concentration of chitosan could offer a base for an optimum modulation in drug dose and its gels are efficient in local treatment of periodontal disease [26].

Similarly, doxycycline nanoliposome slow-release gel was evaluated for therapeutic effects on an established rat model of periodontitis. The biocompatibility of the system was examined by oral perfusion of the sample gel for a prolonged observation. The results reveal that doxycycline nanogel exhibit excellent biocompatibility from weight measure and tissue section evaluation. Furthermore, the NPs-hydrogel composite system demonstrates ability to improve the periodontitis condition of the rats with periodontitis defects [46].

To demonstrate the suitability and effectiveness of nanocomposite in repelling periodontal pathogens, a team of researchers developed a bioactive nanocomposite for Class $\mathrm{V}$ restorations with a combination of protein-repellent and antibacterial capabilities to combat periodontal biofilms. They fabricated resin which consists of ethoxylated bisphenol A dimethacrylate and pyromellitic glycerol dimethacrylate. Then, 2-methacryloyloxyethyl phosphorylcholine (MPC), dimethylaminohexadecyl methacrylate (DMAHDM), and NPs of amorphous calcium phosphate were incorporated into the previously made resin. The mechanical properties of the nanocomposite were similar to those of a commercial composite used in Class V restorations. The composite with 3\% DMAHDM showed a strong antibacterial capability. The researchers concluded that the use of dual agents, i.e., 3\% DMAHDM and 3\% MPC, in the nanocomposite yielded a significant reduction in periodontal microbial biofilm growth, metabolic activity, and polysaccharide production. Hence, this novel nanocomposite is a promising device for Class $\mathrm{V}$ restorations to inhibit periodontal pathogens and for the treatment of periodontitis [47]. 
Nanogels in life-threatening diseases

Nanotechnological innovation in drug delivery is not limited to revolutionizing dental therapeutics alone but extends to many other fields of disease treatment (Table 3). Studies have shown that through nanotechnological techniques, there are possibilities of developing nanogel delivery systems whereby two drugs are loaded in a unit delivery system. Mark Tibbitt of Massachusetts Institute of Technology, USA and coresearchers carried out a study, in which two drugs were loaded in an nanogels. They developed polyethylene glycol-poly lactic acid NPs which were suited to carry hydrophobic small-molecule drug. On the other hand, they made a gel with a cellulose polymer suited to carry hydrophilic drug and then mixed the two components together which resulted in nanogels. They concluded that the research could be a potential model for delivering two different drugs at the same time, for the treatment of certain diseases, such as macular degeneration, cancer, and heart disease [48].

In another study, hydrogel-NPs with thermoresponsive properties were prepared and characterized. Inverse emulsion polymerization method was used in its development by crosslinking of acrylate derivatives of poly (ethylene glycol) and poly (ethylene glycol)-blpoly (propylene glycol)-blpoly (ethylene glycol) copolymers, also

Table 1: Some investigated localized drug delivery system for the treatment of periodontal disease

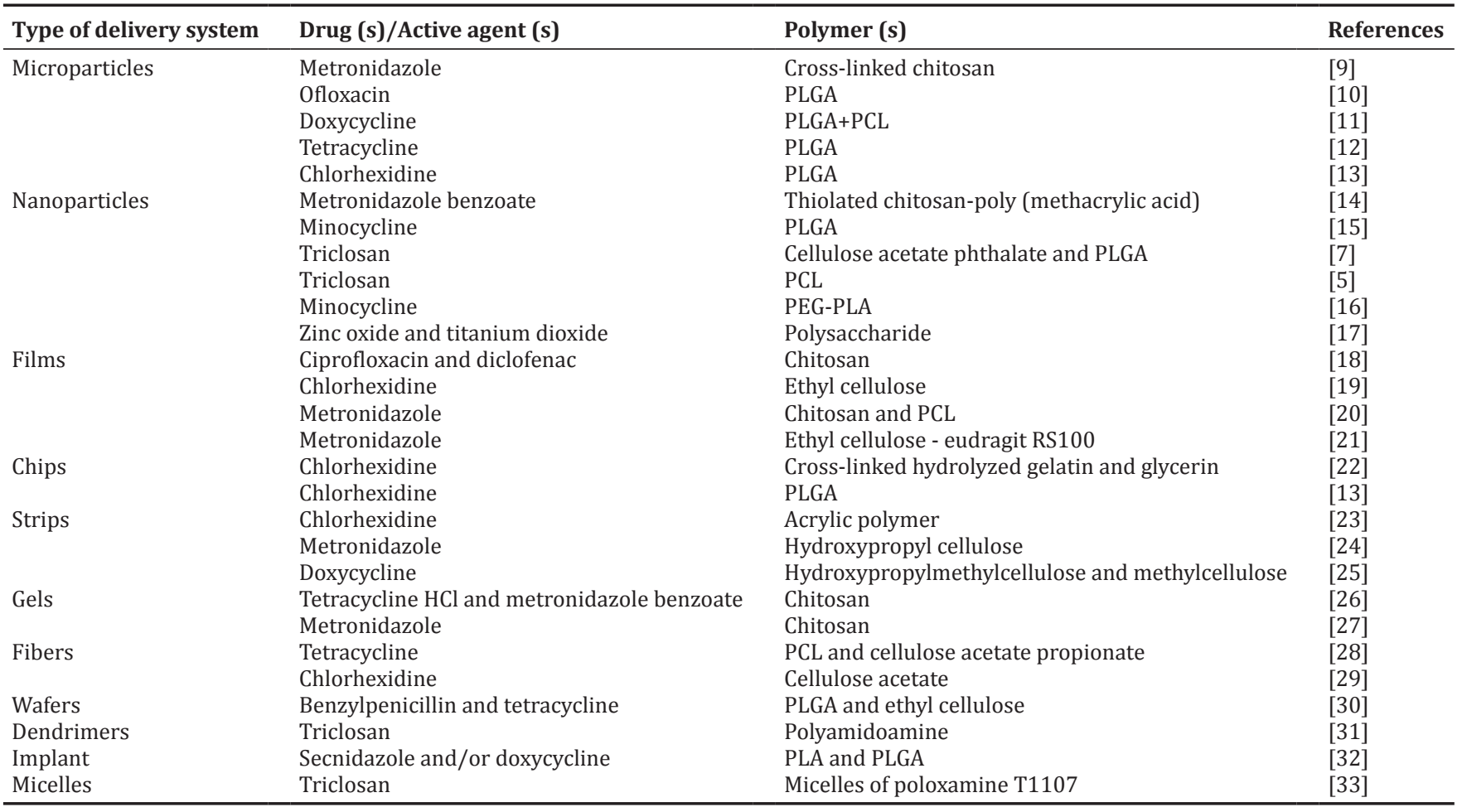

PLGA: Poly (dl-lactic-co-glycolic acid), PLA: Poly lactic acid, PCL: Poly-e-caprolactone, HCl: Hydrochloric acid

Table 2: Important applications of nanogel drug delivery systems [36-40]

- Through the nanogels, multiple drugs can be incorporated and delivered to the targeted site even without chemical cross-linkage. This is highly

desirable in order to maintain the original drug activity

- The nanogel systems can be suitably fabricated for various routes of administration such as oral cavity which include periodontal area,

parenteral, intra-ocular and nasal cavity

- The nanogels provide opportunity for designing both passive and active targeting because of its surface nature and molecular size

- It also possesses high degree of drug loading, extreme high water content (hydrophilicity), flexibility in size as well as surface electrical charge

- Ease of production and handling, availability of raw materials for fabrication, and remarkable biocompatibility

- Nanogels can be formulated with site-specific targeting that can be achieved through linking ligands to the surface of nanoparticles embedded in it and consequently might enhance the solubility and pharmacokinetics of the conjugated drug molecules

Table 3: Summary of some nanoparticles-hydrogel composite drug delivery systems designed for various diseases

\begin{tabular}{|c|c|c|c|c|c|}
\hline System & $\begin{array}{l}\text { Loaded active agent (s)/ } \\
\text { drug (s) }\end{array}$ & Polymer (s) & Method of preparation & $\begin{array}{l}\text { Targeted } \\
\text { disease }(s)\end{array}$ & References \\
\hline Topical nanogels & Acitretin and aloe-emodin & Chitin & Regeneration chemistry method & Psoriasis & {$[51]$} \\
\hline Topical nanogels & Curcumin & Chitin & Controlled regeneration method & Skin cancer & [52] \\
\hline $\begin{array}{l}\text { Self-assembling } \\
\text { nanogels }\end{array}$ & $\begin{array}{l}\text { Antimicrobial peptides } \\
\text { LLKKK18 }\end{array}$ & Hyaluronic acid & Conjugate synthesis & Tuberculosis & {$[53]$} \\
\hline $\begin{array}{l}\text { Redox-responsive } \\
\text { nanogels }\end{array}$ & Doxorubicin & $\begin{array}{l}\text { Oligoethyleneglycol } \\
\text { and pyridyldisulfide }\end{array}$ & Self-crosslinking & Ovarian cancer & [54] \\
\hline
\end{tabular}


known as Pluronics ${ }^{\circledR}$. These synthesized hydrogel NPs were of size range of $100-500 \mathrm{~nm}$ and were found to be stable. The nanogels were fabricated with both hydrophilic and hydrophobic polymers to enable the accommodation of poorly water soluble active agents through hydrophobic interactions, and it has been determined to be potential carriers for drug delivery in cancer treatment [49].

To enhance the oral bioavailability of myricetin (a natural antioxidant flavonol identified to have a variety of therapeutic applications), a novel chitosan-based nanogels were comprehensively developed and evaluated. The formulated myricetin-loaded nanogels were of 100-300 $\mathrm{nm}$ particle sizes range, and it displayed sol-gel transitions when exposed to physiological temperatures. The nanogels swelled in $\mathrm{pH} 6.8$ and $\mathrm{pH} 7.4$ buffers and were easily eroded in acidic buffer solutions (pH 1.2 and $\mathrm{pH} 4.5$ ). The nanogel systems was able to release the loaded drugs moderately through a Fickian mechanism, and the oral bioavailability of myricetin in rats was improved by the developed novel nanogels delivery system [39].

A novel nanogels loaded with an anticancer agent (5-fluorouridine) of poly- $\alpha, \beta$-polyasparthydrazide has been developed by reverse microemulsion technique with redox-responsive release mechanism. The smart nanogels had a NP size $(\sim 200 \mathrm{~nm})$, a polyamino acid structure, a high drug loading of more than $20 \%$. The nanogels was prepared by crossing-link reaction of poly- $\alpha, \beta$-polyasparthydrazide chains and 3,3'-dithiodipropionic acid through redox-responsive chain network. The developed system has good control of drug release profile and it showed excellent redox-responsibility and low cytotoxicity. These confirm it applicability as a potential controlled-release carrier for a redox-responsive tumor-targeting delivery of the anticancer agent 5-fluorouridine [50].

\section{FUTURE DIRECTIONS}

Although the effort toward treating periodontal diseases has led to the advent of some successful delivery devices, there is still need for collective efforts to exploit more benefits in the NPs-based delivery system arena so that effective treatment could be achieved. Fig. 3 classifies various current and future strategies for the treatment of periodontitis. The current approaches suffer from disadvantages such as limited effectiveness, poor biodistribution, short residence time, and lack of selectivity.

Therefore, the development of intra-pocket nanoparticulate delivery system with bioadhesive, biocompatible, and biodegradable polymer(s) that can provide targeted and controlled drug release would be highly desirable. The future research should focus on the use of bioadhesive polymers to enhance the residence time of the periodontal delivery systems. These future approaches should be able to make optimum contact with the mucosal surfaces in the periodontium and should prolong the residence time at the targeted site (i.e., in periodontal

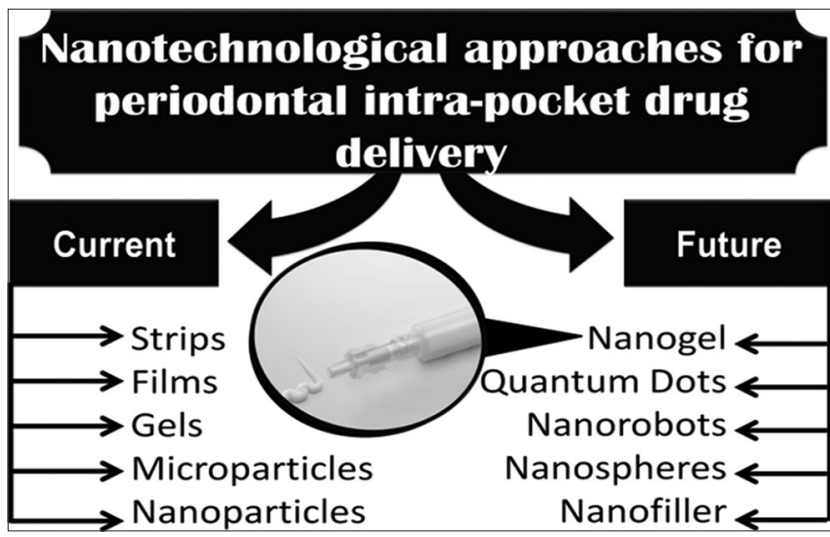

Fig. 3: Classification of various current and future nanoparticulate drug delivery systems for the treatment of periodontitis pocket) and should also intensify contact with the junctional epithelium so as to enhance the epithelial transport of poorly absorbable drugs. The use of non-steroidal anti-inflammatory drugs (NSAIDs) in the treatment of periodontal disease seems to be feasible as research has shown that it may reduce gingivitis and slow progression of the disease [56].

\section{Roles of nanogels in the future}

Nanogels are expected to play a significant role in the future management of periodontitis, owing to its unique potential of achieving multidrug therapy and providing beneficial characteristics of both NPs and that of hydrogel systems, such as bioadhesiveness and biocompatibility (which could be imparted by the polymers) [57], the ability to encapsulate large amounts of drug(s) within its matrix, the high stability in aqueous medium, and the feature of releasing drugs in a controlled manner.

The future nanogels drug delivery innovations are focusing on drug release control based on stimuli responses. These nanogels with the prefix "smart" should be able to sense the changes in its surroundings and regulate the drug release profile accordingly. The invention of such systems may have profound potential applications, especially for drugs with narrow index or those with severe side effects [37]. Smart nanogels should be able to suitably react to certain environmental stimuli with instantaneous feedback when such specific stimuli signals are detected from stimulus. Studies have already proved that nanogels generally demonstrated rapid response compared to the conventional hydrogel particles [58]. These features will further qualify the use of nanogels in designing suitable drug delivery systems for various ailments including periodontitis. The initial investigations show that nanogels can changes it physical, chemical, electrical, and/or optical properties in response to a specific stimulus in its surrounding.

Some of the smart nanogel systems that are drawing attention are:

- Temperature-responsive nanogels

- Photo-responsive-based nanogels

- pH-responsive nanogels

- Magnetically-responsive nanogels

- Ultrasound-responsive nanogels

- Redox-responsive nanogels

There is no doubt that nanogel drug delivery systems will play a vital role in future drug delivery systems not only for periodontitis but for a lot of other diseases that affect the human race. Investigations are underway for more exploitation, and some researchers had already reported applicability of these novel systems in the treatment of various illnesses as summarized in Table 3. The features and flexibility which nanogels provided has led to the opening of new horizons for nanotechnologybased drug delivery systems for the treatment of diseases, hence, it will be of great significance in the future nanoparticulate drug delivery systems.

\section{CONCLUSION}

The recent developments in nanomaterials and nanotechnology have provided promising opportunities for the effective management of periodontitis. Some of the excellent results and rewards achievable from these novel approaches are the use of bioadhesive polymers to achieve prolonged drug release, the increment of intra-pocket drug penetration, the enhancement of mechanical properties using chemical crosslinkers, and the possibility of loading multiple drugs in a unit delivery system. Furthermore, the advent of smart-nanogel innovations whereby drug release may be control by stimuli responses gives additional promising opportunities in achieving optimum therapeutic effect. The future utilization of these advantages will significantly improve dental care. The coformulation of nanoparticles-hydrogel composite showed additional advantages compared to simple NPs or hydrogels as local intra-pocket drug delivery systems. These rewards will pave the way for further research opportunities in drug delivery system development, thus advancing dental therapeutics. 


\section{ACKNOWLEDGMENT}

Mr. Nafiu Aminu wish to gratefully acknowledge the tremendous support provided by Universiti Sains Malaysia, Penang through a USM fellowship. The support is highly appreciated.

\section{REFERENCES}

1. Pihlstrom BL, Michalowicz BS, Johnson NW. Periodontal diseases. Lancet 2005;366(9499):1809-20.

2. Jain N, Jain GK, Javed S, Iqbal Z, Talegaonkar S, Ahmad FJ, et al. Recent approaches for the treatment of periodontitis. Drug Discov Today 2008;13(21-22):932-43.

3. Page RC, Schroeder HE. Pathogenesis of inflammatory periodontal disease. A summary of current work. Lab Invest 1976;34(3):235-49.

4. Savage A, Eaton KA, Moles DR, Needleman I. A systematic review of definitions of periodontitis and methods that have been used to identify this disease. J Clin Periodontol 2009;36(6):458-67.

5. Aminu N, Baboota S, Pramod K, Singh M, Dang S, Ansari SH, et al. Development and evaluation of triclosan loaded poly- $\varepsilon$-caprolactone nanoparticulate system for the treatment of periodontal infections. J Nanopart Res 2013;15(11):2075.

6. Wilczewska AZ, Niemirowicz K, Markiewicz KH, Car H. Nanoparticles as drug delivery systems. Pharmacol Rep 2012;64(5):1020-37.

7. Piñón-Segundo E, Ganem-Quintanar A, Alonso-Pérez V, Quintanar-Guerrero D. Preparation and characterization of triclosan nanoparticles for periodontal treatment. Int J Pharm 2005;294(12):217-32.

8. Wu H, Yu G, Pan L, Liu N, McDowell MT, Bao Z, et al. Stable Li-ion battery anodes by in-situ polymerization of conducting hydrogel to conformally coat silicon nanoparticles. Nat Commun 2013;4:1943.

9. Pichayakorn W, Boonme P. Evaluation of cross-linked chitosan microparticles containing metronidazole for periodontitis treatment. Mater Sci Eng C Mater Biol Appl 2013;33(3):1197-202.

10. Jamal T, Rahman MA, Mirza MA, Panda AK, Talegaonkar S, Iqbal Z. Formulation, antimicrobial and toxicity evaluation of bioceramic based ofloxacin loaded biodegradable microspheres for periodontal infection. Curr Drug Deliv 2012;9(5):515-26.

11. Shanmuganathan S, Shanumugasundaram N, Adhirajan N, Lakshmi TS, Babu M. Preparation and characterization of chitosan microspheres for doxycycline delivery. Carbohydr Polym 2008;73(2):201-11.

12. Esposito E, Cortesi R, Cervellati F, Menegatti E, Nastruzzi C. Biodegradable microparticles for sustained delivery of tetracycline to the periodontal pocket: Formulatory and drug release studies. J Microencapsul 1997;14(2):175-87.

13. Yue IC, Poff J, Cortés ME, Sinisterra RD, Faris CB, Hildgen P, et al. A novel polymeric chlorhexidine delivery device for the treatment of periodontal disease. Biomaterials 2004;25(17):3743-50.

14. Saboktakin MR, Tabatabaie RM, Maharramov A, Ramazanov MA. Development and in vitro evaluation of thiolated chitosan - Poly(methacrylic acid) nanoparticles as a local mucoadhesive delivery system. Int J Biol Macromol 2011;48(3):403-7.

15. Kashi TS, Eskandarion S, Esfandyari-Manesh M, Marashi SM, Samadi N, Fatemi SM, et al. Improved drug loading and antibacterial activity of minocycline-loaded PLGA nanoparticles prepared by solid/ oil/water ion pairing method. Int J Nanomedicine 2012;7:221-34

16. Yao W, Xu P, Pang Z, Zhao J, Chai Z, Li X, et al. Local delivery of minocycline-loaded PEG-PLA nanoparticles for the enhanced treatment of periodontitis in dogs. Int J Nanomedicine 2014;9:3963-70.

17. Khan ST, Al-Khedhairy AA, Musarrat J. ZnO and TiO2 nanoparticles as novel antimicrobial agents for oral hygiene: A review. J Nanopart Res 2015;17(6):276

18. Ahmed M, Harish N, Charyulu R, Prabhu P. Formulation of chitosan-based ciprofloxacin and diclofenac film for periodontitis therapy. Trop J Pharm Res 2009;8(1):33-41

19. Hirschfeld Z, Friedman M, Golomb G, Ben-Yaacov D. New sustained release dosage form of chlorhexidine for dental use: Use for plaque control in partial denture wearers. J Oral Rehabil 1984;11(5):477-82.

20. El-Kamel AH, Ashri LY, Alsarra IA. Micromatricial metronidazole benzoate film as a local mucoadhesive delivery system for treatment of periodontal diseases. AAPS PharmSciTech 2007;8(3):E75.

21. Vineetha VC, Mathews MM. Development and evaluation of dental films containing an antibacterial agent for the treatment of periodontitis. Int J Pharm Pharm Sci 2015;7(3):52-9.

22. Mizrak T, Güncü GN, Caglayan F, Balci TA, Aktar GS, Ipek F. Effect of a controlled-release chlorhexidine chip on clinical and microbiological parameters and prostaglandin E2 levels in gingival crevicular fluid. J Periodontol 2006;77(3):437-43.

23. Addy M, Langeroudi M. Comparison of the immediate effects on the sub-gingival microflora of acrylic strips containing $40 \%$ chlorhexidine, metronidazole or tetracycline. J Clin Periodontol 1984;11(6):379-86.

24. Wade WG, Moran J, Morgan JR, Newcombe R, Addy M. The effects of antimicrobial acrylic strips on the subgingival microflora in chronic periodontitis. J Clin Periodontol 1992;19(2):127-34

25. Taner IL, Ozcan G, Doğanay $T$, Iscanolu M, Taplamacioğlu B, Gültekin SE, et al. Comparison of the antibacterial effects on subgingival microflora of two different resorbable base materials containing doxycycline. J Nihon Univ Sch Dent 1994;36(3):183-90.

26. Popa L, Ghica MV, Dinu-Pîrvu CE. Periodontal chitosan-gels designed for improved local intra-pocket drug delivery. Farmacia 2013;61(2):240-50.

27. Akncbay H, Senel S, Ay ZY. Application of chitosan gel in the treatment of chronic periodontitis. J Biomed Mater Res B Appl Biomater 2007;80(5):290-6.

28. Raheja I, Kohli K, Drabu S. Periodontal drug delivery system containing antimicrobial agents. Int J Pharm Pharm Sci 2013;5(3):11-6.

29. Coventry J, Newman HN. Experimental use of a slow release device employing chlorhexidine gluconate in areas of acute periodontal inflammation. J Clin Periodontol 1982;9(2):129-33.

30. Bromberg LE, Buxton DK, Friden PM. Novel periodontal drug delivery system for treatment of periodontitis. J Control Release 2001;71(3):251-9.

31. Gardiner J, Freeman S, Leach M, Green A, Alcock J, D'Emanuele A. PAMAM dendrimers for the delivery of the antibacterial Triclosan. J Enzyme Inhib Med Chem 2008;23(5):623-8.

32. Gad HA, El-Nabarawi MA, Abd El-Hady SS. Formulation and evaluation of PLA and PLGA in situ implants containing secnidazole and/or doxycycline for treatment of periodontitis. AAPS PharmSciTech 2008;9(3):878-84

33. Chiappetta DA, Degrossi J, Teves S, D'Aquino M, Bregni C, Sosnik A. Triclosan-loaded poloxamine micelles for enhanced topical antibacterial activity against biofilm. Eur J Pharm Biopharm 2008;69(2):535-45.

34. Bhavikatti SK, Bhardwaj S, Prabhuji ML. Current applications of nanotechnology in dentistry: A review. Gen Dent 2014;62(4):72-7.

35. Bhardwaj A, Bhardwaj A, Misuriya A, Maroli S, Manjula S, Singh AK. Nanotechnology in dentistry: Present and future. J Int Oral Health 2014;6(1):121-6

36. Bidwai VS, Rane PS, Talele SG, Jadhav A. Nanogel: A versatile targeted drug delivery system. Indo Am J Pharm Res 2015;5(12):3905-13.

37. Hamidi M, Rostamizadeh K, Shahbazi MA. Hydrogel nanoparticles in drug delivery. In: Intelligent Nanomaterials. Hoboken, NJ, USA: John Wiley \& Sons, Inc.; 2012. p. 583-624.

38. Pedrosa SS, Gonçalves C, David L, Gama M. A novel crosslinked hyaluronic acid nanogel for drug delivery. Macromol Biosci 2014;14(11):1556-68.

39. Yao Y, Xia M, Wang H, Li G, Shen $\mathrm{H}, \mathrm{Ji}$ G, et al. Preparation and evaluation of chitosan-based nanogels/gels for oral delivery of myricetin. Eur J Pharm Sci 2016;91:144-53.

40. Sarangi MK, Padhi S. Solid lipid nanoparticles - A review. J Crit Rev 2016;3(3):5-12.

41. Bako J, Szepesi M, Veres AJ, Cserhati C, Borbely ZM, Hegedus C, et al. Synthesis of biocompatible nanocomposite hydrogels as a local drug delivery system. Colloid Polym Sci 2008;286(3):357-63.

42. Nagatomo K, Komaki M, Sekiya I, Sakaguchi Y, Noguchi K, Oda S, et al. Stem cell properties of human periodontal ligament cells. J Periodontal Res 2006;41(4):303-10.

43. Hasegawa M, Yamato M, Kikuchi A, Okano T, Ishikawa I. Human periodontal ligament cell sheets can regenerate periodontal ligament tissue in an athymic rat model. Tissue Eng 2005;11(3-4):469-78.

44. Akizuki T, Oda S, Komaki M, Tsuchioka H, Kawakatsu N, Kikuchi A, et al. Application of periodontal ligament cell sheet for periodontal regeneration: A pilot study in beagle dogs. J Periodontal Res 2005;40(3):245-51.

45. Fukui T, Kobayashi H, Hasegawa U, Nagasawa T, Akiyoshi K, Ishikawa I. Intracellular delivery of nanogel-quantum dot hybrid nanoparticles into human periodontal ligament cells. Drug Metab Lett 2007;1(2):131-5

46. Jin HL, Wu W, Shu R. Doxycycline nano-liposome slow-release gel improves rat periodontitis. Shanghai Kou Qiang Yi Xue 2010;19(5):508-11.

47. Wang L, Xie $\mathrm{X}$, Imazato $\mathrm{S}$, Weir $\mathrm{MD}$, Reynolds $\mathrm{MA}, \mathrm{Xu} \mathrm{HH}$. A protein-repellent and antibacterial nanocomposite for Class-V restorations to inhibit periodontitis-related pathogens. Mater Sci Eng C 
Mater Biol Appl 2016;67:702-10.

48. Trafton A. New Nanogel for Drug Delivery. Massachusetts Institute of Technology News, 2015. Available from: http://www.news.mit. edu/2015/self-healing-nanogel-drug-delivery-0219. [Last cited on 2015 Nov 18].

49. Missirlis D. Development of a Novel Drug Delivery System Based on Polymeric, Thermoresponsive, Hydrogel Nanoparticles. Ecole Polytechnique Fédérale de Lausanne (EPFL), 2005. Available from: http://www.infoscience.epfl.ch/record/54793.

50. Guo J, Wang Y, Wang J, Zheng X, Chang D, Wang S, et al. A novel nanogel delivery of poly- $\alpha, \beta$-polyasparthydrazide by reverse microemulsion and its redox-responsive release of 5-fluorouridine. Asian J Pharm Sci 2016. doi: 10.1016/j.ajps.2016.05.008.

51. Divya G, Panonnummal R, Gupta S, Jayakumar R, Sabitha M. Acitretin and aloe-emodin loaded chitin nanogel for the treatment of psoriasis. Eur J Pharm Biopharm 2016;107:97-109.

52. Mangalathillam S, Rejinold NS, Nair A, Lakshmanan VK, Nair SV, Jayakumar R. Curcumin loaded chitin nanogels for skin cancer treatment via the transdermal route. Nanoscale 2012;4(1):239-50.

53. Silva JP, Gonçalves C, Costa C, Sousa J, Silva-Gomes R, Castro AG, et al. Delivery of LLKKK18 loaded into self-assembling hyaluronic acid nanogel for tuberculosis treatment. J Control Release 2016;235:112-24.

54. Asadi $\mathrm{H}$, Khoee $\mathrm{S}$. Dual responsive nanogels for intracellular doxorubicin delivery. Int J Pharm 2016;511(1):424-35.

55. Zabihi F, Wieczorek S, Dimde M, Hedtrich S, Börner HG, Haag R. Intradermal drug delivery by nanogel-peptide conjugates; Specific and efficient transport of temoporfin. J Control Release 2016. pii: S0168-365930468-0.

56. Harvey S, Zieve D. Periodontitis: Other Treatments. The New York Times, 2013. Available from: http://www.nytimes.com/health/ guides/disease/periodontitis/other-treatments.html. [Last cited on 2016 Feb 10].

57. Munot NM, Gujar KN. Orodental delivery systems: An overview. Int J Pharm Pharm Sci 2013;5(3):74-83.

58. Hamidi M, Azadi A, Rafiei P. Hydrogel nanoparticles in drug delivery. Adv Drug Deliv Rev 2008;60(15):1638-49. 